\title{
Assessment of post-operative pain in cats: a case study on veterinary students of Universiti Putra Malaysia
}

\begin{abstract}
The ability to assess and control pain is listed as one of the desired Day One competencies among veterinary graduates. As such, a study was conducted to examine the current status and effectiveness of a video-based training module on the attitude toward and knowledge of pain assessment in cats among fourth- and final-year veterinary students of Universiti Putra Malaysia (UPM) in January of 2013. A total of 92 students participated in this study, resulting in a response rate of $60.1 \%$. Upon completion of a pre-training survey, the respondents undertook an interactive video-based presentation, followed by a post-training survey. The majority of the students $(96.7 \%)$ agreed on the importance of pain management. Before the training, many $(76.1 \%)$ disagreed that they had received adequate training, while 53.3\% were not confident in their pain-recognition skills. After training, their knowledge and confidence in pain assessment increased. Responses to the survey were not associated with differences in gender, level of study, or field of interest. Students were found to have mistaken some physiologic parameters as good pain indicators after ovariohysterectomy. Their assessment of three standardized video cases revealed that they could recognize prominent signs of pain but failed to identify changes in behavior that were more subtle. Refinement to the training module is required to address the above deficiencies.
\end{abstract}

Keyword: Cats; Pain; Training; Veterinary student; Video cases 